\title{
Interpolation-based Parameterized Model Order Reduction of Delayed Systems
}

\author{
Francesco Ferranti, Member, IEEE, Michel Nakhla, Fellow, IEEE, Giulio Antonini, Senior Member, IEEE, Tom \\ Dhaene, Senior Member, IEEE, Luc Knockaert, Senior Member, IEEE, and Albert E. Ruehli, Life Fellow, IEEE
}

\begin{abstract}
D electromagnetic methods are fundamental tools for the analysis and design of high-speed systems. These methods often generate large systems of equations and model order reduction (MOR) methods are used to reduce such a high complexity. When the geometric dimensions become electrically large or signal waveform rise times decrease, time delays must be included in the modeling.

Design space optimization and exploration are usually performed during a typical design process that consequently requires repeated simulations for different design parameter values. Efficient performing of these design activities call for parameterized model order reduction (PMOR) methods, which are able to reduce large systems of equations with respect to frequency and other design parameters of the circuit, such as layout or substrate features.

We propose a novel PMOR method for neutral delayed differential systems, which is based on an efficient and reliable combination of univariate model order reduction methods, a procedure to find scaling and frequency shifting coefficients and positive interpolation schemes. The proposed scaling and frequency shifting coefficients enhance and improve the modeling capability of standard positive interpolation schemes and allow accurate modeling of highly dynamic systems with a limited amount of initial univariate models in the design space. The proposed method is able to provide parameterized reduced order models passive by construction over the design space of interest. Pertinent numerical examples validate the proposed PMOR approach.
\end{abstract}

Index Terms-Delayed systems, Interpolation, Parameterized model order reduction (PMOR), Partial Element Equivalent Circuit method (PEEC).

\section{INTRODUCTION}

Complex high-speed systems require 3-D electromagnetic (EM) methods [1]-[3] as analysis and design tools. Large

Manuscript received June 2011.

Francesco Ferranti, Tom Dhaene and Luc Knockaert are with the Department of Information Technology, Internet Based Communication Networks and Services (IBCN), Ghent University-IBBT, Gaston Crommenlaan 8 Bus 201, B-9050 Gent, Belgium, email: \{francesco.ferranti, tom.dhaene, luc.knockaert\}@ugent.be.

M. Nakhla is with the Department of Electronics, Carleton University, Ottawa, ON K1S 5B6, Canada, e-mail: msn@doe.carleton.ca.

Giulio Antonini is with the UAq EMC Laboratory, Dipartimento di Ingegneria Elettrica e dell'Informazione, Università degli Studi dell'Aquila, Via G. Gronchi 18,67100, L'Aquila, Italy, e-mail: giulio.antonini@univaq.it.

Albert E. Ruehli is an Emeritus of the IBM T. J. Watson Research Center, Yorktown Heights, NY 10598, USA and an Adjunct Professor at the Missouri University of S\&T in Rolla, e-mail: albert.ruehli@gmail.com.

This work was supported by the Research Foundation Flanders (FWO) and by the Italian Ministry of University (MIUR) under a Program for the Development of Research of National Interest, (PRIN grant n. 20089J4SM9002). systems of equations are usually generated by the use of these methods and model order reduction (MOR) techniques are crucial to reduce the complexity of EM models and the computational cost of the simulations, while retaining the important physical features of the original system [4]-[7]. Over the last years, the development of methods to build reduced order models (ROMs) of EM systems has been intensively investigated, with applications to interconnects, vias and high-speed packages [8]-[11]. Among all EM methods, the Partial Element Equivalent Circuit (PEEC) method [2] has been found particularly useful for modeling PCBs, interconnects, and power systems. The PEEC method uses a circuit interpretation of the Electric Field Integral Equation (EFIE) [12] and it is especially suitable to problems involving both electromagnetic fields and circuits [2], [13], [14]. PEEC equivalent circuits are usually connected to nonlinear circuit devices such as drivers and receivers using a time domain circuit simulator (e.g. SPICE [15]). Complex systems can result in PEEC models where the number of circuit elements can be in the tens of thousands, and therefore the inclusion of these PEEC models directly into a circuit simulator may be computationally intractable.

When signal waveform rise times decrease and the corresponding frequency content increases or the geometric dimensions become electrically large, time delays must be taken into account and included in the modeling. A PEEC formulation that includes delay elements, called $\tau$ PEEC method [16], becomes necessary and leads to systems of neutral delayed differential equations (NDDE) [17] with constant coefficients and constant delay times in the time domain and to complex algebraic systems of equations with frequency-dependent matrices in the frequency domain. Simply using quasi-static PEEC models can result in significant errors and artifacts in the modeling [18].

Over the years, some techniques for the reduction of NDDE systems have been proposed [19]-[24]. In [21], an equivalent first order system is computed by means of a Taylor expansion, and then MOR Krylov subspace methods [6], [7] are applied. The reduction process does not preserve the NDDE formulation. In [24], an equivalent first order system is computed using a single point Taylor expansion [21] and a corresponding orthogonal projection matrix is computed using a block Arnoldi algorithm [7]. Then, an orthogonal projection matrix for the original NDDE system is extracted and a reduced NDDE system is obtained. All these previous techniques cannot efficiently handle the reduction of electri- 
cally large structures, where delays among coupled elements cannot be neglected or easily approximated by rational basis functions. Recently, a novel MOR method for NDDE systems with large delays has been proposed [25]. It is based on an adaptive multipoint expansion and MOR of equivalent first order systems [24].

Traditional MOR techniques perform model reduction only with respect to frequency. However, during the circuit synthesis of large-scale digital or analog applications, it is also important to predict the response of the circuit under study as a function of design parameters, such as geometrical and substrate features. A typical design process includes optimization and design space exploration, and thus requires repeated simulations for different design parameter values. Such design activities call for parameterized model order reduction (PMOR) methods that can reduce large systems of equations with respect to frequency and other design parameters of the circuit, such as geometrical layout or substrate characteristics.

Several PMOR techniques have been proposed over the years. Concerning rational systems, multiparameter momentmatching methods presented in [26]-[28] use a subspace projection approach to provide parameterized ROMs, while similar approaches are described in [29]-[31] for NDDE systems. However, the structure of such methods may present some computational problems, and the resulting reduced models usually suffer from oversize when the number of moments to match is high, either because high accuracy (order) is required or because the number of parameters is large. The selection of the multidimensional expansion points and the number of multiparameter moments need to be addressed in these methods. The technique presented in [32] combines traditional passivitypreserving model order reduction methods and interpolation schemes based on a class of positive interpolation operators. A PMOR method based on a parameterization process of matrices generated by EM methods and projection subspaces is proposed in [33]. Overall passivity of parameterized ROMs is guaranteed over the design space of interest in [32], [33].

This paper proposes a PMOR method for NDDE systems, which is based on an efficient and reliable combination of univariate model order reduction methods, a procedure to find scaling and frequency shifting coefficients and positive interpolation schemes [34]. The PMOR method proposed in this paper starts by computing a set of reduced order $\tau$ PEEC models ( $\tau$ ROMs) using the MOR algorithm [25] for different design parameters values. We note that the MOR method [25] is able to perform reduction only with respect to frequency. Then, a set of scaling and frequency shifting coefficients is computed for the set of $\tau$ PEEC reduced models, which are finally interpolated to build a parameterized reduced model. In [32], a set of reduced models is interpolated to build a parameterized reduced model using standard positive interpolation schemes. The new proposed scaling and frequency shifting coefficients enhance and improve the modeling capability of standard positive interpolation schemes [32] and allow accurate modeling of highly dynamic systems with a limited amount of initial univariate models in the design space. The new proposed method does not have to deal with multiparameter moment computations and related issues. The expansion points are chosen only along the frequency axis for the $\tau$ ROMs using an adaptive algorithm [25]. Under the assumptions that the original $\tau$ PEEC models are passive and the MOR method used to provide the $\tau$ ROMs is passivitypreserving, the proposed PMOR method is able to build parameterized reduced models that are passive over the entire design space of interest.

The paper is organized as follows. Section II describes the modified nodal analysis (MNA) equations of the $\tau$ PEEC method. Section III describes the proposed PMOR method for NDDE systems. Finally, some pertinent numerical examples based on $\tau$ PEEC models validate the proposed technique in Section IV.

\section{II. $\tau$ PEEC FORMULATION}

The PEEC method [2] stems from the integral equation form of Maxwell's equations. With respect to other EM methods, it is worth pointing out its capability to provide a circuit interpretation of the EFIE equation, thus allowing it to handle complex problems involving both circuits and electromagnetic fields.

In the standard approach, volumes and surfaces are discretized into elementary regions, hexahedra and patches respectively [16] over which the current and charge densities are expanded into a series of basis functions.

Following the standard Galerkin's testing procedure, topological elements, namely nodes and branches, are generated and electrical lumped elements are identified modeling both the magnetic and electric field coupling.

Conductors are modeled by their ohmic resistance, while dielectrics require modeling the polarization charge due to the dielectric polarization [35]. Magnetic and electric field coupling are modeled by partial inductances and coefficients of potential, respectively.

An example of $\tau$ PEEC circuit for a conductor elementary cell is illustrated, in the Laplace domain, in Fig. 1 where the current controlled voltage sources $s L_{p, i j} I_{j}$ and the charge controlled current sources $s Q_{i}$ model the magnetic and electric field couplings, respectively.

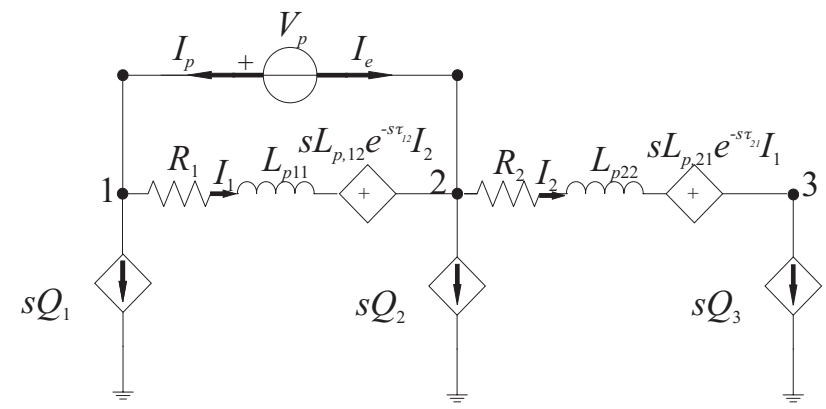

Fig. 1. Illustration of $\tau$ PEEC circuit electrical quantities for a conductor elementary cell.

Let us assume that the meshing process of conductors and dielectrics has generated $n_{i}$ volume cells where currents flow and $n_{n}$ surface cells where charge is located; the resultant 
number of elementary cells of conductors and dielectrics is $n_{c}$ and $n_{d}$, respectively and that of electrical nodes is $n_{n}$. Furthermore, let us assume to be interested in generating an admittance representation $\mathbf{Y}(s)$ having $n_{p}$ output currents $\mathbf{i}_{p}(t)$ under voltage excitation $\mathbf{v}_{p}(t)$. If the MNA approach [36] is used, the global number of unknowns is $n_{u}=n_{i}+n_{d}+n_{n}+n_{p}$ and an admittance representation of the PEEC circuit is obtained [25]

$$
\begin{aligned}
\mathbf{C}(\boldsymbol{\tau}) \frac{d \mathbf{x}(t)}{d t} & =-\mathbf{G}(\boldsymbol{\tau}) \mathbf{x}(t)+\mathbf{B u}(t) \\
\mathbf{i}_{p}(t) & =\mathbf{L}^{T} \mathbf{x}(t)
\end{aligned}
$$

where $\mathbf{x}(t) \in \Re^{n_{u} \times 1}$ and $\boldsymbol{\tau} \in \Re^{n_{\tau} \times 1}$ contains all $n_{\tau}$ delays $\left\{\tau_{k}\right\}_{k=1}^{n_{\tau}}$, which denote the center-to-center delay matrices for the magnetic and electric field coupling. Since this is an $n_{p^{-}}$ port formulation, whereby the only sources are the voltage sources at the $n_{p}$-port nodes, $\mathbf{B}=\mathbf{L}$ where $\mathbf{B} \in \Re^{n_{u} \times n_{p}}$. Each delayed entry of matrices $\mathbf{C}(\boldsymbol{\tau})$ and $\mathbf{G}(\boldsymbol{\tau})$ act as a delay operator for the corresponding entry of vector $\mathbf{x}(t)$. Hence, (1) can be rewritten in the Laplace domain as:

$$
\begin{aligned}
s \mathbf{C}(s) \mathbf{X}(s) & =-\mathbf{G}(s) \mathbf{X}(s)+\mathbf{B V}_{p}(s) \\
\mathbf{I}_{p}(s) & =\mathbf{L}^{T} \mathbf{X}(s) \\
\mathbf{C}(s) & =\mathbf{C}_{0}+\sum_{k=1}^{n_{\tau}} \mathbf{C}_{k} e^{-s \tau_{k}} \\
\mathbf{G}(s) & =\mathbf{G}_{0}+\sum_{k=1}^{n_{\tau}} \mathbf{G}_{k} e^{-s \tau_{k}}
\end{aligned}
$$

which corresponds to the admittance transfer function

$$
\mathbf{Y}(s)=\mathbf{L}^{T}(s \mathbf{C}(s)+\mathbf{G}(s))^{-1} \mathbf{B}
$$

The matrices $\mathbf{C}_{0}, \mathbf{G}_{0}$ denote the quasi-static contribution.

\section{Parameterized Model Order Reduction}

Considering the influence of the design parameters $\boldsymbol{g}=$ $\left(g^{(1)}, \ldots, g^{(\mathrm{N})}\right)$ such as layout and substrate features, the MNA formulation (1a)-(1b) becomes

$$
\begin{aligned}
\mathbf{C}(\boldsymbol{\tau}, \boldsymbol{g}) \frac{d \mathbf{x}(t, \boldsymbol{g})}{d t} & =-\mathbf{G}(\boldsymbol{\tau}, \boldsymbol{g}) \mathbf{x}(t, \boldsymbol{g})+\mathbf{B}(\boldsymbol{g}) \mathbf{u}(t)(7 \mathrm{a}) \\
\mathbf{i}_{p}(t, \boldsymbol{g}) & =\mathbf{L}(\boldsymbol{g})^{T} \mathbf{x}(t, \boldsymbol{g})
\end{aligned}
$$

while (6) becomes

$$
\mathbf{Y}(s, \boldsymbol{g})=\mathbf{L}(\boldsymbol{g})^{T}(s \mathbf{C}(s, \boldsymbol{g})+\mathbf{G}(s, \boldsymbol{g}))^{-1} \mathbf{B}(\boldsymbol{g})
$$

In this section, we describe a PMOR algorithm that is able to include, in addition to frequency, $\mathrm{N}$ design parameters $\boldsymbol{g}=$ $\left(g^{(1)}, \ldots, g^{(\mathrm{N})}\right)$ in the reduced order model. The main objective of this PMOR method is to accurately approximate the original scalable system (having a high complexity) with a reduced scalable system (having a low complexity) by capturing the behaviour of the original system with respect to frequency and other design parameters.

The proposed algorithm guarantees passivity of the parameterized reduced models over the entire design space of interest, under the assumptions that the original $\tau$ PEEC models are passive and the MOR method used to provide the $\tau$ ROMs is passivity-preserving. A flowchart that describes the different steps of the proposed PMOR method is shown in Fig. 2.

$$
\text { Compute } \tau \operatorname{ROMs} \mathbf{R}\left(s, \mathbf{g}_{k}^{\Omega_{i}}\right)
$$

in the estimation design space grid

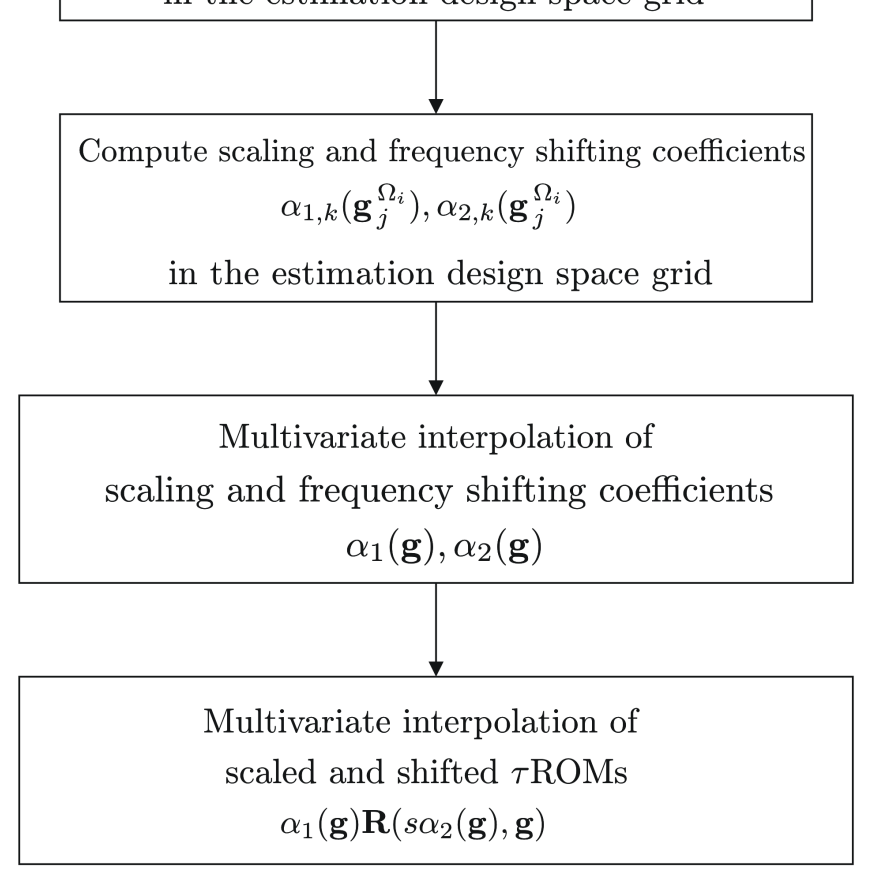

Fig. 2. Flowchart of the proposed PMOR method.

\section{A. $\tau R O M s$}

The proposed PMOR technique starts by computing a set of reduced order models of the PEEC admittance matrix $\mathbf{Y}(s, \boldsymbol{g})=\mathbf{L}^{T}(\boldsymbol{g})(s \mathbf{C}(s, \boldsymbol{g})+\mathbf{G}(s, \boldsymbol{g}))^{-1} \mathbf{B}(\boldsymbol{g})$, called $\tau$ ROMs, using the MOR method described in [25] for a set of points in the design space, which we call estimation design space grid. The design space $\mathcal{D}(\boldsymbol{g})$ is considered as the parameter space $\mathcal{P}(s, \boldsymbol{g})$ without frequency. The parameter space $\mathcal{P}(s, \boldsymbol{g})$ contains all parameters $(s, \boldsymbol{g})$. If the parameter space is $\mathrm{N}$-dimensional, the design space is $(\mathrm{N}-1)$-dimensional. Two design space grids are used in the modeling process: an estimation grid and a validation grid. The first grid is utilized to build the $\tau$ ROMs. The second grid is utilized to assess the capability of parameterized reduced models of describing the system under study in a set of points of the design space previously not used for the construction of the $\tau$ ROMs. To clarify the use of these two design space grids, we show in Fig. 3 a possible estimation and validation design space grid in the case of two design parameters $\boldsymbol{g}=\left(g^{(1)}, g^{(2)}\right)$. A $\tau$ ROM is built for each red cross (x) point in the design space. The set of $\tau$ ROMs is interpolated, as explained in what follows, to build a parameterized reduced model that is evaluated and compared with original $\tau$ PEEC models related to the blue circle (o) design space points. We note that these blue circle (o) points are not used for the generation of the $\tau$ ROMs. 


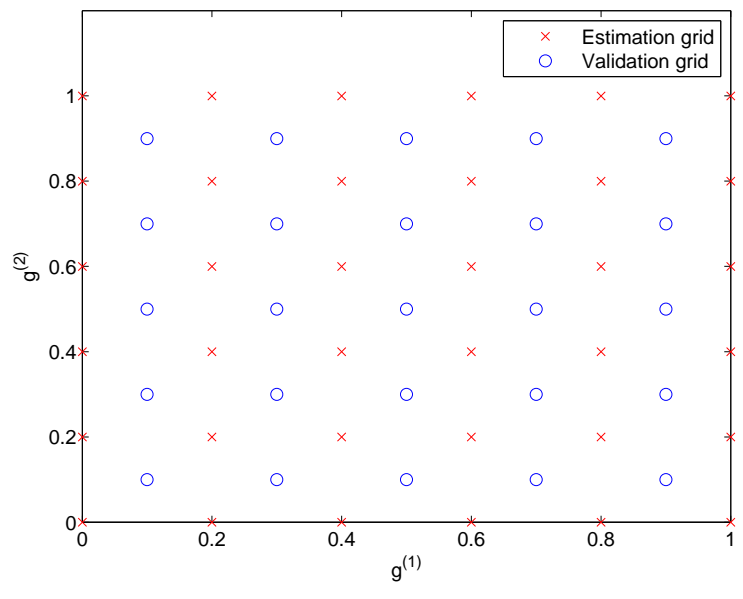

Fig. 3. An example of estimation and validation design space grid.

$\mathrm{N}$-dimensional and scattered design space grids can also be treated by the proposed PMOR technique that does not impose any constraint on the number of design parameters and the distribution of $\tau$ ROMs in the design space.

\section{B. Scaling and Frequency Shifting Coefficients}

After the computation of the $\tau$ ROMs, the next step is combining them together and building a multivariate representation $\mathbf{R}(s, \boldsymbol{g})$. The design space is divided into cells using hyperrectangles (regular grids) [37] or simplices (regular and scattered grids) [38]. We note that the proposed PMOR technique can handle regular and scattered design space grids.

Once the design space is divided into cells, a local parameterized model is associated to every cell that is a subdomain of the entire design space. We indicate a cell region of the design space as $\Omega_{i}, i=1, \ldots, P$ (shown in Fig. 4) and the corresponding vertices as $\boldsymbol{g}_{k}^{\Omega_{i}}, k=1, \ldots, Q$.

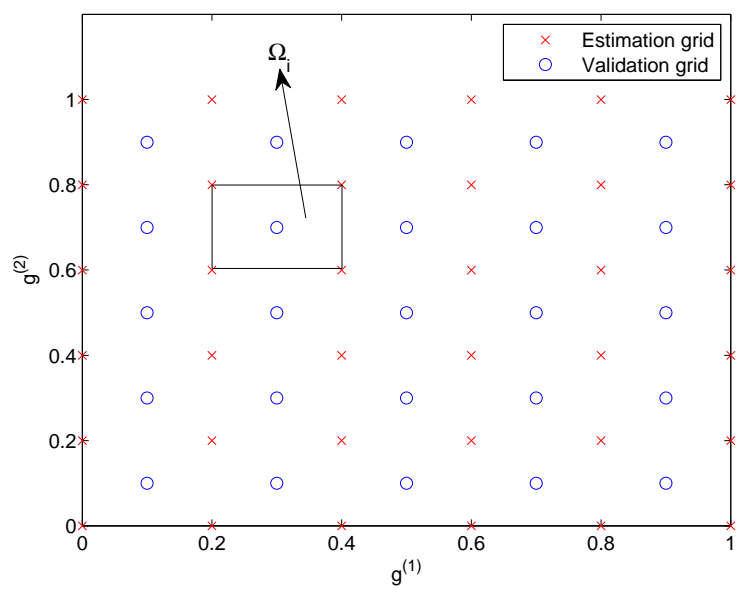

Fig. 4. An example of a design space cell $\Omega_{i}$.

We note that each vertex corresponds to a $\tau$ ROM $\mathbf{R}\left(s, \boldsymbol{g}_{k}^{\Omega_{i}}\right)$. For each cell, an optimization procedure is used to find the scaling and frequency shifting system coefficients that make each vertex an accurate approximant of the other cell vertices. For each vertex $\mathbf{R}\left(s, \boldsymbol{g}_{k}^{\Omega_{i}}\right)$, a set of scaling $\alpha_{1, k}\left(\boldsymbol{g}_{j}^{\Omega_{i}}\right), j=1, \ldots, Q$ and frequency shifting $\alpha_{2, k}\left(\boldsymbol{g}_{j}^{\Omega_{i}}\right), j=1, \ldots, Q$ real coefficients are found, such that

$$
\begin{gathered}
\alpha_{1, k}\left(\boldsymbol{g}_{j}^{\Omega_{i}}\right) \mathbf{R}\left(s \alpha_{2, k}\left(\boldsymbol{g}_{j}^{\Omega_{i}}\right), \boldsymbol{g}_{k}^{\Omega_{i}}\right) \simeq \mathbf{R}\left(s, \boldsymbol{g}_{j}^{\Omega_{i}}\right), j \neq k \\
\alpha_{1, k}\left(\boldsymbol{g}_{j}^{\Omega_{i}}\right)=\alpha_{2, k}\left(\boldsymbol{g}_{j}^{\Omega_{i}}\right)=1, j=k
\end{gathered}
$$

This optimization problem can be solved using, for example, the Matlab [39] routines fmincon and fminsearchbnd with $\alpha_{1, k}\left(\mathbf{g}_{j}^{\Omega_{i}}\right)=1, \alpha_{2, k}\left(\mathbf{g}_{j}^{\Omega_{i}}\right)=1, j=1, \ldots, Q, j \neq k$ as initial guess. These routines are able to impose some constraints on the optimized coefficients, which is important to guarantee the passivity of parameterized reduced order models as explained in what follows. If the response of the system under modeling needs to be computed in a specific design space point $\widehat{\boldsymbol{g}}$, a subdomain that contains $\widehat{\boldsymbol{g}}$ is to be found. For each vertex $\tau$ ROM of the found subdomain, the corresponding sets of scaling and frequency shifting coefficients $\alpha_{1, k}\left(\boldsymbol{g}_{j}^{\Omega_{i}}\right), \alpha_{2, k}\left(\boldsymbol{g}_{j}^{\Omega_{i}}\right)$ are interpolated in $\widehat{\boldsymbol{g}}$ and a model $\widehat{\alpha}_{1, k} \mathbf{R}\left(s \widehat{\alpha}_{2, k}, \boldsymbol{g}_{k}^{\Omega_{i}}\right)$ is built, where $\widehat{\alpha}_{1, k}=\alpha_{1, k}(\widehat{\boldsymbol{g}})$ and $\widehat{\alpha}_{2, k}=\alpha_{2, k}(\widehat{\boldsymbol{g}})$. Finally, the set of modified $\tau$ ROMs $\widehat{\alpha}_{1, k} \mathbf{R}\left(s \widehat{\alpha}_{2, k}, \boldsymbol{g}_{k}^{\Omega_{i}}\right), k=1, \ldots, Q$, is interpolated at an input/output level as described in [32]. We note that if a generic $\tau \operatorname{ROM} \mathbf{R}\left(s, \boldsymbol{g}_{k}^{\Omega_{i}}\right)$ has the admittance representation

$$
\mathbf{L}\left(\boldsymbol{g}_{k}^{\Omega_{i}}\right)^{T}\left(s \mathbf{C}\left(s, \boldsymbol{g}_{k}^{\Omega_{i}}\right)+\mathbf{G}\left(s, \boldsymbol{g}_{k}^{\Omega_{i}}\right)\right)^{-1} \mathbf{B}\left(\boldsymbol{g}_{k}^{\Omega_{i}}\right)
$$

then a corresponding scaled and frequency shifted version $\widehat{\alpha}_{1, k} \mathbf{R}\left(s \widehat{\alpha}_{2, k}, \boldsymbol{g}_{k}^{\Omega_{i}}\right)$ has the admittance representation

$$
\widetilde{\mathbf{L}}\left(\boldsymbol{g}_{k}^{\Omega_{i}}\right)^{T}\left(s \widetilde{\mathbf{C}}\left(s, \boldsymbol{g}_{k}^{\Omega_{i}}\right)+\widetilde{\mathbf{G}}\left(s, \boldsymbol{g}_{k}^{\Omega_{i}}\right)\right)^{-1} \widetilde{\mathbf{B}}\left(\boldsymbol{g}_{k}^{\Omega_{i}}\right)
$$

with

$$
\begin{aligned}
\widetilde{\mathbf{C}}\left(s, \boldsymbol{g}_{k}^{\Omega_{i}}\right) & =\mathbf{C}\left(s \widehat{\alpha}_{2, k}, \boldsymbol{g}_{k}^{\Omega_{i}}\right) \\
\widetilde{\mathbf{G}}\left(s, \boldsymbol{g}_{k}^{\Omega_{i}}\right) & =\left(\widehat{\alpha}_{2, k}\right)^{-1} \mathbf{G}\left(s \widehat{\alpha}_{2, k}, \boldsymbol{g}_{k}^{\Omega_{i}}\right) \\
\widetilde{\mathbf{B}}\left(\boldsymbol{g}_{k}^{\Omega_{i}}\right) & =\mathbf{B}\left(\boldsymbol{g}_{k}^{\Omega_{i}}\right) \\
\widetilde{\mathbf{L}}\left(\boldsymbol{g}_{k}^{\Omega_{i}}\right) & =\widehat{\alpha}_{1, k}\left(\widehat{\alpha}_{2, k}\right)^{-1} \mathbf{L}\left(\boldsymbol{g}_{k}^{\Omega_{i}}\right)
\end{aligned}
$$

The scaling coefficients $\alpha_{1, k}\left(\boldsymbol{g}_{j}^{\Omega_{i}}\right)$ can be extended to scaling matrices $\boldsymbol{\Gamma}_{1, k}\left(\boldsymbol{g}_{j}^{\Omega_{i}}\right)$ such that a modified $\tau$ ROM $\widehat{\boldsymbol{\Gamma}}_{1, k}^{T} \mathbf{R}\left(s \widehat{\alpha}_{2, k}, \boldsymbol{g}_{k}^{\Omega_{i}}\right) \widehat{\boldsymbol{\Gamma}}_{1, k}$ is obtained with

$$
\begin{aligned}
\widetilde{\mathbf{C}}\left(s, \boldsymbol{g}_{k}^{\Omega_{i}}\right) & =\mathbf{C}\left(s \widehat{\alpha}_{2, k}, \boldsymbol{g}_{k}^{\Omega_{i}}\right) \\
\widetilde{\mathbf{G}}\left(s, \boldsymbol{g}_{k}^{\Omega_{i}}\right) & =\left(\widehat{\alpha}_{2, k}\right)^{-1} \mathbf{G}\left(s \widehat{\alpha}_{2, k}, \boldsymbol{g}_{k}^{\Omega_{i}}\right) \\
\widetilde{\mathbf{B}}\left(\boldsymbol{g}_{k}^{\Omega_{i}}\right) & =\mathbf{B}\left(\boldsymbol{g}_{k}^{\Omega_{i}}\right) \widehat{\boldsymbol{\Gamma}}_{1, k} \\
\widetilde{\mathbf{L}}\left(\boldsymbol{g}_{k}^{\Omega_{i}}\right) & =\left(\widehat{\alpha}_{2, k}\right)^{-1} \mathbf{L}\left(\boldsymbol{g}_{k}^{\Omega_{i}}\right) \widehat{\boldsymbol{\Gamma}}_{1, k}
\end{aligned}
$$


where superscript "T" is the transpose operator. Even if the use of scaling matrices can provide more flexibility in the optimization step, we verified that the use of simple scaling coefficients leads to good results in terms of accuracy, while making the optimization faster and independent from the number of ports. In the numerical examples, scalar scaling and frequency shifting coefficients will be used.

\section{Multivariate Interpolation}

Passivity is crucial when the reduced model is utilized in a circuit simulator for transient analysis. Passive systems cannot generate more energy than they absorb through their electrical ports. When the system is terminated on any arbitrary passive loads, none of them will cause the system to become unstable. A linear network described by admittance matrix $\mathbf{Y}(s)$ is passive if [40]:

1) $\mathbf{Y}\left(s^{*}\right)=\mathbf{Y}^{*}(s)$ for all $s$, where " $*$ " is the complex conjugate operator

2) $\mathbf{Y}(s)$ is analytic in $\Re e(s)>0$.

3) $\mathbf{Y}(s)$ is a positive-real matrix, i.e. : $\mathbf{z}^{* T}\left(\mathbf{Y}^{T}\left(s^{*}\right)+\mathbf{Y}(s)\right) \mathbf{z} \geq 0 ; \forall s: \Re e(s)>0$ and any arbitrary vector $\mathbf{z}$.

Similar results are valid for a linear network described by impedance matrix $\mathbf{Z}(s)$. The interpolated scaling and frequency shifting real coefficients $\alpha_{1, k}(\boldsymbol{g}), \alpha_{2, k}(\boldsymbol{g})$ have to satisfy the following conditions

$$
\begin{aligned}
& \alpha_{1, k}(\boldsymbol{g}) \geq 0 \\
& \alpha_{2, k}(\boldsymbol{g})>0
\end{aligned}
$$

to preserve the passivity of the corresponding scaled and frequency shifted $\tau$ ROM. If scaling matrices $\boldsymbol{\Gamma}_{1, k}(\boldsymbol{g})$ are used instead of scaling coefficients $\alpha_{1, k}(\boldsymbol{g})$, no particular condition has to be satisfied by $\boldsymbol{\Gamma}_{1, k}(\boldsymbol{g})$. Multivariate interpolation schemes based on a class of positive interpolation operators [41] are used to parameterize $\alpha_{1, k}(\boldsymbol{g}), \alpha_{2, k}(\boldsymbol{g})$. These schemes are able to guarantee the passivity of each scaled and frequency shifted $\tau$ ROM by satisfying the properties (15a)-(15b). The same positive multivariate interpolation schemes are used to interpolate the set of modified $\tau$ ROMs $\widehat{\alpha}_{1, k} \mathbf{R}\left(s \widehat{\alpha}_{2, k}, \boldsymbol{g}_{k}^{\Omega_{i}}\right), \quad k=1, \ldots, Q$ at an input/output level, which results in a parameterized reduced model passive over the entire design space.

Multivariate interpolation can be realized by means of tensor product [42] or tessellation [38] methods. Any interpolation scheme based on a class of positive interpolation operators can be used.

In the bivariate case $(s, g)$, each interpolated function $\mathbf{T}(g)$, being in turn $\alpha_{1, k}(g), \alpha_{2, k}(g), \mathbf{R}(s, g)$, can be written as

$$
\mathbf{T}(g)=\sum_{k=1}^{K_{1}} \mathbf{T}_{g_{k}} \ell_{k}(g)
$$

where $K_{1}$ represents the number of $\tau$ ROMs vertices of a specific subdomain and each interpolation kernel $\ell_{k}(g)$ is a scalar function satisfying the following constraints

$$
\begin{gathered}
\ell_{k}(g) \geq 0, \\
\ell_{k}\left(g_{i}\right)=\delta_{k, i},
\end{gathered}
$$

A possible choice is to select $\ell_{k}(g)$ as in piecewise linear interpolation.

In the general multivariate case, multivariate interpolation methods that belong to the general class of positive interpolation operators can be used, e.g., the piecewise multilinear and multivariate simplicial methods [37]. We note that the interpolation kernel functions of these methods only depend on the design space grid points and their computation does not require the solution of a linear system to impose an interpolation constraint. These positive interpolation schemes have been already used in [32]. In the proposed new PMOR technique, a powerful novelty is introduced by the interpolation process of a set of scaling and frequency shifting system coefficients, which increase the modeling capability of the proposed algorithm with respect to [32], where the interpolation process were only applied to the ROMs treated as input-output systems.

\section{Passivity Preserving Interpolation}

Under the assumptions that the original $\tau$ PEEC models are passive and the MOR method used to provide the $\tau$ ROMs is passivity-preserving, the proposed PMOR method is able to build parameterized reduced models that are passive over the entire design space of interest.

A scaling coefficient $\alpha_{1}$ or matrix $\boldsymbol{\Gamma}_{1}$ is applied at the input/output level of the system, while a frequency shifting coefficient $\alpha_{2}$ is a compression or expansion term for the Laplace variable $s$. Considering admittance and impedance representations, if $\alpha_{1}, \alpha_{2}$ satisfy (15a)-(15b), passivity is preserved when these coefficients are applied to a passive system. It is straightforward to prove that if a nonnegative scalar coefficient is applied to a passive system, it preserves the three passivity conditions. A positive frequency shifting coefficient is a compression or expansion term for the Laplace variable $s$ and therefore if a system is passive in the $s$-domain, it is also passive in a compressed or expanded $s$-domain.

Once a set of scaled and frequency shifted $\tau$ ROMs $\widehat{\alpha}_{1, k} \mathbf{R}\left(s \widehat{\alpha}_{2, k}, \boldsymbol{g}^{\Omega_{i}}\right), k=1, \ldots, Q$, which are passive, is built for each cell in the estimation design space grid, the next step of the proposed PMOR method is focused on combining together these $\tau$ ROMs by a multivariate interpolation scheme to obtain a parameterized $\operatorname{ROM} \mathbf{R}(s, \boldsymbol{g})$ with overall passivity. Conditions 1)-2) are preserved in (16) and corresponding multivariate extensions, as they are weighted sums with real nonnegative weights of systems respecting these two conditions. Concerning Condition 3), we refer to the following theorem [43]:

Theorem 1: Any nonnegative linear combination of positive real matrices is a positive real matrix.

Since (16) and the multivariate extensions are weighted sums with real nonnegative weights of passive systems, condition 3) is satisfied by construction over the entire design space of interest. We have proven that all the three passivity conditions for admittance (and impedance) representations are preserved in our PMOR algorithm. 


\section{E. Method complexity and parallelization}

As shown in Fig. 2, the proposed method is composed of four steps with a related complexity:

1) the construction of the $\tau$ ROMs is performed using the MOR method [25];

2) the computation of scaling and frequency shifting coefficients is performed using optimization routines. We recall that using scalar scaling and frequency shifting coefficients leads to a good accuracy of parameterized ROMs, while keeping the optimization process computationally cheap and fast;

3) the multivariate interpolation of scaling and frequency shifting coefficients uses positive local interpolation methods with interpolation kernel functions that only depend on the estimation grid points;

4) comments similar to those of the previous point are valid for the multivariate interpolation of scaled and shifted $\tau$ ROMs. We recall that the estimation design space grid is divided into cells using hyperrectangles (regular grids) [37] or simplices (regular and scattered grids) [38]. Each cell is defined by a specific number of vertices that are used to perform interpolation in the cell itself.

All four steps of the proposed technique have a certain degree of parallelism:

1) since each $\tau \mathrm{ROM}$ is independent from the others, the construction of a single $\tau$ ROM can be performed in parallel with the others;

2) the computation of scaling and frequency shifting coefficients can be parallelized cell by cell;

3) a parallelization cell by cell is also feasible for the multivariate interpolation of scaling and frequency shifting coefficients;

4) comments similar to those of the previous point are valid for the multivariate interpolation of scaled and shifted $\tau$ ROMs.

\section{NUMERICAL RESULTS}

This section presents two numerical examples that validate the proposed PMOR method. Let us define the weighted RMSerror as:

$$
\begin{aligned}
& \operatorname{Err}(\boldsymbol{g})= \\
& =\sqrt{\frac{\sum_{i=1}^{N_{i} N_{o}} \sum_{k=1}^{K_{s}}\left|w_{Y_{i}}\left(s_{k}, \boldsymbol{g}\right)\left(Y_{r, i}\left(s_{k}, \boldsymbol{g}\right)-Y_{i}\left(s_{k}, \boldsymbol{g}\right)\right)\right|^{2}}{N_{i} N_{o} K_{s}}}
\end{aligned}
$$

with

$$
w_{Y_{i}}(s, \boldsymbol{g})=\left|\left(Y_{i}(s, \boldsymbol{g})\right)^{-1}\right|
$$

where $N_{i}, N_{o}$ are the number of input and output system ports and $K_{s}$ is the number of frequency samples. The worst case RMS-error over the validation grid is chosen to assess the accuracy and the quality of parameterized ROMs

$$
\begin{gathered}
\boldsymbol{g}_{\text {max }}=\underset{\boldsymbol{g}}{\operatorname{argmax}} \operatorname{Err}(\boldsymbol{g}), \boldsymbol{g} \in \text { validation grid } \\
\operatorname{Err}_{\max }=\operatorname{Err}\left(\boldsymbol{g}_{\text {max }}\right)
\end{gathered}
$$

and it is used in the numerical examples. The proposed PMOR algorithm was implemented in Matlab R2009A [39] and all experiments were carried out on Windows platform equipped with Intel Core2 Extreme CPU Q9300 2.53GHz and 8GB RAM.

\section{A. Three-port microstrip power-divider circuit}

A three-port microstrip power-divider circuit [44], [45] has been modeled in this example. The structure is shown in Fig. 5. The dimensions of the circuit are $[20,20,0.5] \mathrm{mm}$ in the $[x, y, z]$ directions and the width of the microstrips is equal to $0.8 \mathrm{~mm}$. The relative dielectric constant is equal to $\epsilon_{r}=2.2$. A bivariate $\tau \mathrm{ROM}$ is built as a function of the length $L$ in addition to frequency. Their corresponding ranges are shown in Table I.

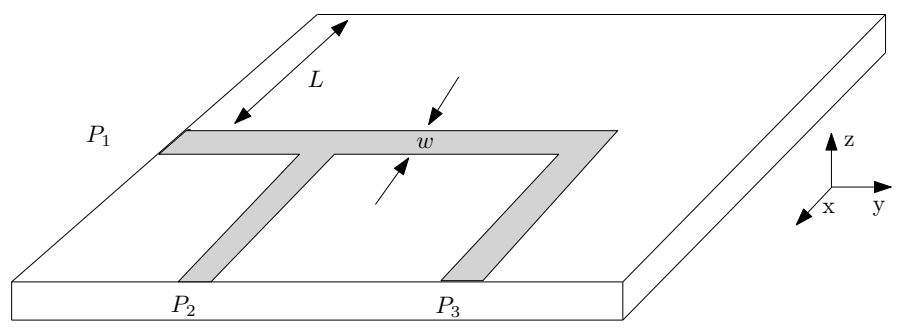

Fig. 5. Structure of the three-port microstrip power-divider circuit.

TABLE I

PARAMETERS OF THE THREE-PORT MICROSTRIP POWER-DIVIDER CIRCUIT.

\begin{tabular}{|l|c|c|}
\hline Parameter & Min & Max \\
\hline Frequency $($ freq $)$ & $100 \mathrm{kHz}$ & $10 \mathrm{GHz}$ \\
Length $(L)$ & $6 \mathrm{~mm}$ & $10 \mathrm{~mm}$ \\
\hline
\end{tabular}

The $\tau$ PEEC method is used to compute the $\mathbf{C}, \mathbf{G}, \mathbf{B}, \mathbf{L}$ matrices in (1a)-(1b) for 11 values of $L$. Then, we have built reduced models for 6 values of $L$ by means of the MOR algorithm described in [25]. Table II shows the order and the number of delays of these $6 \tau$ PEEC models and corresponding $\tau$ ROMs.

TABLE II

(ORDER,DELAYS) OF $\tau$ PEEC MODELS AND $\tau$ ROMS.

\begin{tabular}{|c|c|c|}
\hline & $\tau$ PEEC models & $\tau$ ROMs \\
\hline$L_{1}$ & $(2667,732)$ & $(60,732)$ \\
\hline$L_{2}$ & $(2667,730)$ & $(60,730)$ \\
\hline$L_{3}$ & $(2667,727)$ & $(60,727)$ \\
\hline$L_{4}$ & $(2667,730)$ & $(60,730)$ \\
\hline$L_{5}$ & $(2667,742)$ & $(48,742)$ \\
\hline$L_{6}$ & $(2667,737)$ & $(60,737)$ \\
\hline
\end{tabular}


A bivariate $\tau$ ROM is obtained using the proposed PMOR method with the piecewise linear interpolation scheme. Fig. 6 shows the magnitude of the parameterized reduced model of $\mathbf{Y}_{11}(s, L)$. Fig. 7 shows the magnitude of $\mathbf{Y}_{11}(s, L)$ and $\mathbf{Y}_{12}(s, L)$ for the length values $L=\{6.4,8,9.6\} \mathrm{mm}$. These specific $L$ values have not been used in the $\tau$ ROMs generation process, nevertheless an excellent agreement between reduced and PEEC models can be observed. The worst case RMSerror defined in (22) is equal to 0.08. As clearly seen, the parameterized reduced model captures very accurately the behavior of the system.

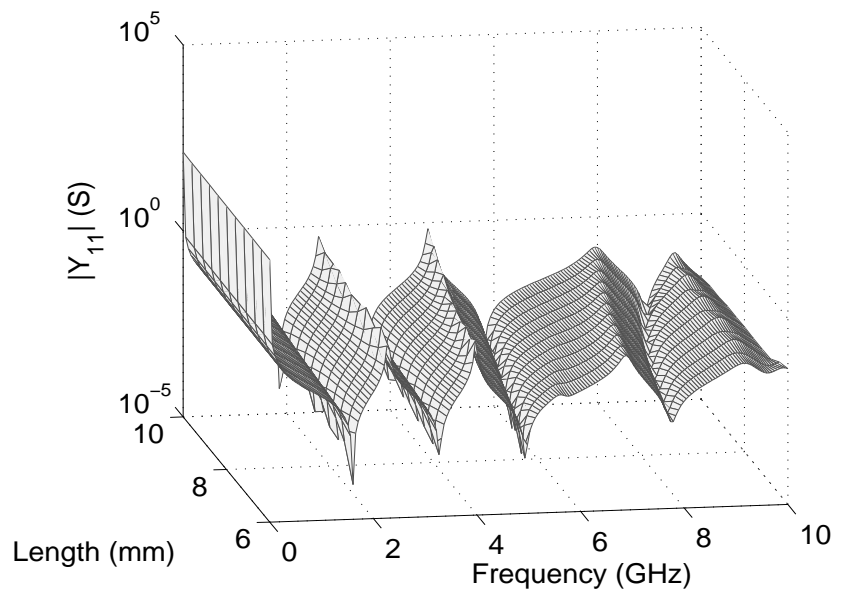

Fig. 6. Magnitude of the bivariate $\tau$ ROM of $\mathbf{Y}_{11}(s, L)$.

\section{B. Three bends}

Three bends over a ground plane with differential ports have been modeled in this example. The structure is shown in Fig. 8. The width of the bends and the distance of the bends from the ground plane are equal to $1 \mathrm{~mm}$. A trivariate $\tau$ ROM is built as a function of the length $L$ and the spacing between the conductors $S$ in addition to frequency. Their corresponding ranges are shown in Table III.

TABLE III

PARAMETERS OF THE THREE BENDS.

\begin{tabular}{|l|c|c|}
\hline Parameter & Min & Max \\
\hline Frequency $($ freq) & $100 \mathrm{kHz}$ & $10 \mathrm{GHz}$ \\
Length $(L)$ & $1 \mathrm{~cm}$ & $1.3 \mathrm{~cm}$ \\
Spacing $(S)$ & $2.5 \mathrm{~mm}$ & $3 \mathrm{~mm}$ \\
\hline
\end{tabular}

The $\tau$ PEEC method is used to compute the $\mathbf{C}, \mathbf{G}, \mathbf{B}, \mathbf{L}$ matrices in (1a)-(1b) over the estimation grid (6 values of $L$ and 5 values of $S$ ) and the validation grid (5 values of $L$ and 4 values of $S$ ), as shown in Fig. 3. Then, we have built $\tau$ ROMs in the estimation grid by means of the MOR algorithm described in [25]. Table IV shows the order and the number of delays of these $30 \tau$ PEEC models and corresponding $\tau$ ROMs. A trivariate $\tau$ ROM is built by means of the presented PMOR approach with the piecewise multilinear interpolation scheme. Fig. 9 shows the magnitude of the parameterized reduced
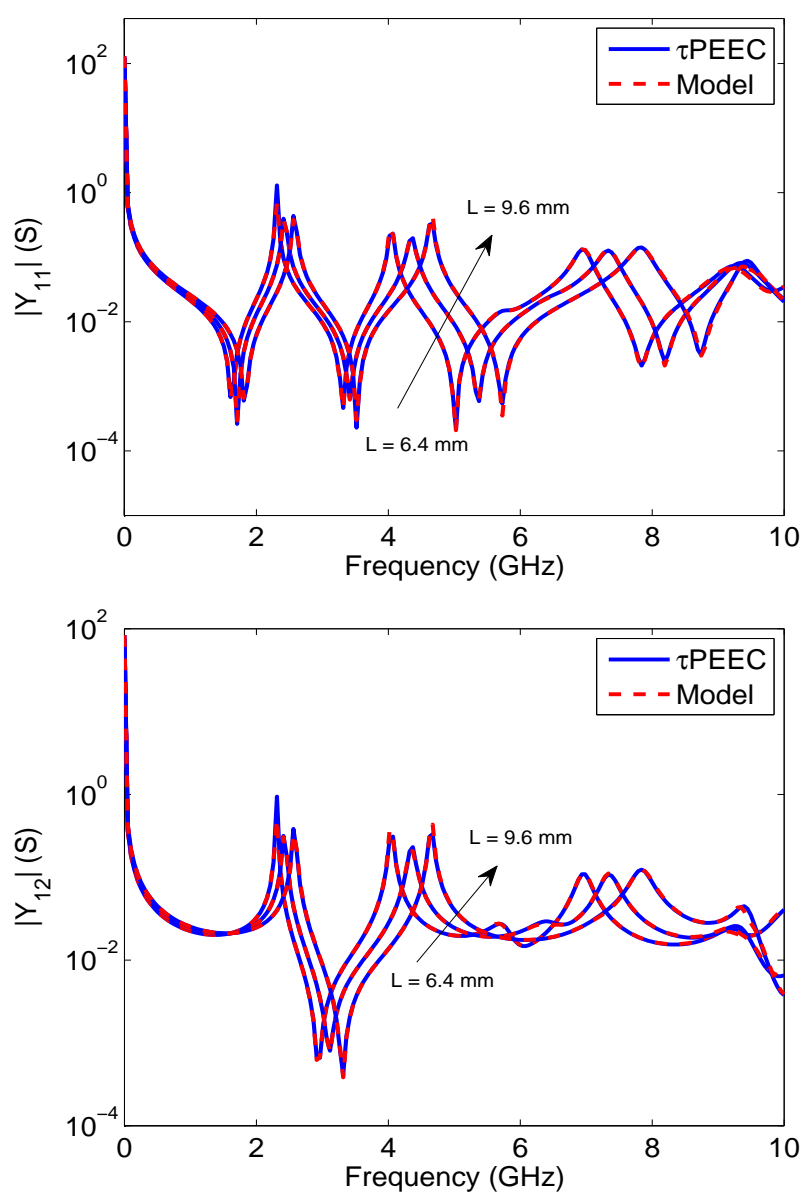

Fig. 7. Magnitude of the bivariate $\tau$ ROMs of $\mathbf{Y}_{11}(s, L)$ and $\mathbf{Y}_{12}(s, L)$ $(L=\{6.4,8,9.6\} \mathrm{mm})$.

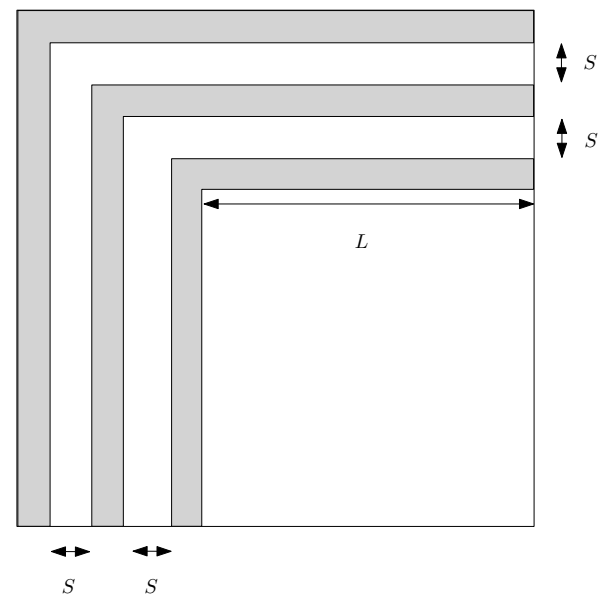

Fig. 8. Structure of the three bends.

model of $\mathbf{Y}_{11}(s, L, S)$ for the spacing values $S=\{2.56,2.97\}$ $\mathrm{mm}$. Fig. 10 shows the magnitude of $\mathbf{Y}_{11}(s, L, S)$ for the length and spacing values $L=\{1.03,1.15 .1 .27\} \mathrm{mm}, S=$ $2.69 \mathrm{~mm}$ and of $\mathbf{Y}_{14}(s, L)$ for the length and spacing values $L=1.15 \mathrm{~mm}, S=\{2.56,2.94\} \mathrm{mm}$. Even if these specific $L$ and $S$ values have not been used in the $\tau$ ROMs generation 
TABLE IV

(ORDER,DELAYS) OF $\tau$ PEEC MODELS AND $\tau$ ROMS.

\begin{tabular}{|c|c|c|}
\hline & $\tau$ PEEC models & $\tau$ ROMs \\
\hline$\left(L_{1}, S_{1}\right)$ & $(2124,510)$ & $(64,510)$ \\
\hline$\left(L_{2}, S_{1}\right)$ & $(2124,508)$ & $(64,508)$ \\
\hline$\left(L_{3}, S_{1}\right)$ & $(2124,510)$ & $(64,510)$ \\
\hline$\left(L_{4}, S_{1}\right)$ & $(2124,511)$ & $(64,511)$ \\
\hline$\left(L_{5}, S_{1}\right)$ & $(2124,517)$ & $(64,517)$ \\
\hline$\left(L_{6}, S_{1}\right)$ & $(2124,519)$ & $(64,519)$ \\
\hline$\left(L_{1}, S_{2}\right)$ & $(2124,509)$ & $(64,509)$ \\
\hline$\left(L_{2}, S_{2}\right)$ & $(2124,511)$ & $(64,511)$ \\
\hline$\left(L_{3}, S_{2}\right)$ & $(2124,513)$ & $(64,513)$ \\
\hline$\left(L_{4}, S_{2}\right)$ & $(2124,512)$ & $(64,512)$ \\
\hline$\left(L_{5}, S_{2}\right)$ & $(2124,519)$ & $(64,519)$ \\
\hline$\left(L_{6}, S_{2}\right)$ & $(2124,520)$ & $(80,520)$ \\
\hline$\left(L_{1}, S_{3}\right)$ & $(2124,511)$ & $(64,511)$ \\
\hline$\left(L_{2}, S_{3}\right)$ & $(2124,514)$ & $(64,514)$ \\
\hline$\left(L_{3}, S_{3}\right)$ & $(2124,518)$ & $(64,518)$ \\
\hline$\left(L_{4}, S_{3}\right)$ & $(2124,515)$ & $(64,515)$ \\
\hline$\left(L_{5}, S_{3}\right)$ & $(2124,518)$ & $(64,518)$ \\
\hline$\left(L_{6}, S_{3}\right)$ & $(2124,522)$ & $(64,522)$ \\
\hline$\left(L_{1}, S_{4}\right)$ & $(2124,511)$ & $(64,511)$ \\
\hline$\left(L_{2}, S_{4}\right)$ & $(2124,512)$ & $(64,512)$ \\
\hline$\left(L_{3}, S_{4}\right)$ & $(2124,519)$ & $(64,519)$ \\
\hline$\left(L_{4}, S_{4}\right)$ & $(2124,519)$ & $(64,519)$ \\
\hline$\left(L_{5}, S_{4}\right)$ & $(2124,519)$ & $(64,519)$ \\
\hline$\left(L_{6}, S_{4}\right)$ & $(2124,527)$ & $(64,527)$ \\
\hline$\left(L_{1}, S_{5}\right)$ & $(2124,510)$ & $(64,510)$ \\
\hline$\left(L_{2}, S_{5}\right)$ & $(2124,513)$ & $(64,513)$ \\
\hline$\left(L_{3}, S_{5}\right)$ & $(2124,518)$ & $(64,518)$ \\
\hline$\left(L_{4}, S_{5}\right)$ & $(2124,520)$ & $(64,520)$ \\
\hline$\left(L_{5}, S_{5}\right)$ & $(2124,519)$ & $(80,519)$ \\
\hline$\left(L_{6}, S_{5}\right)$ & $(2124,524)$ & $(64,524)$ \\
\hline
\end{tabular}

process, the model accurately describes the system under study in these design space points. The worst case RMSerror defined in (22) is equal to 0.065. The parameterized reduced model is able to accurately describe the parameterized behavior of the system.

\section{Conclusions}

We have presented a new PMOR technique applicable to NDDE systems. It is based on an efficient and reliable combination of univariate model order reduction methods, a procedure to find scaling and frequency shifting coefficients and positive interpolation schemes. An innovative passivitypreserving interpolation of reduced systems at an input-output level is proposed, which allows accurately modeling of highly dynamic systems. The proposed method does not have to deal with multiparameter moment computation and related issues. The expansion points are chosen only along the frequency axis using an adaptive algorithm. Under the assumptions that the original $\tau$ PEEC models are passive and the MOR method used to provide the $\tau$ ROMs is passivity-preserving, the proposed PMOR method is able to build parameterized reduced models that are passive over the entire design space of interest. Numerical examples have validated the proposed PMOR approach on practical application cases, showing that it is able to build very accurate parameterized $\tau$ ROMs of dynamic EM systems.
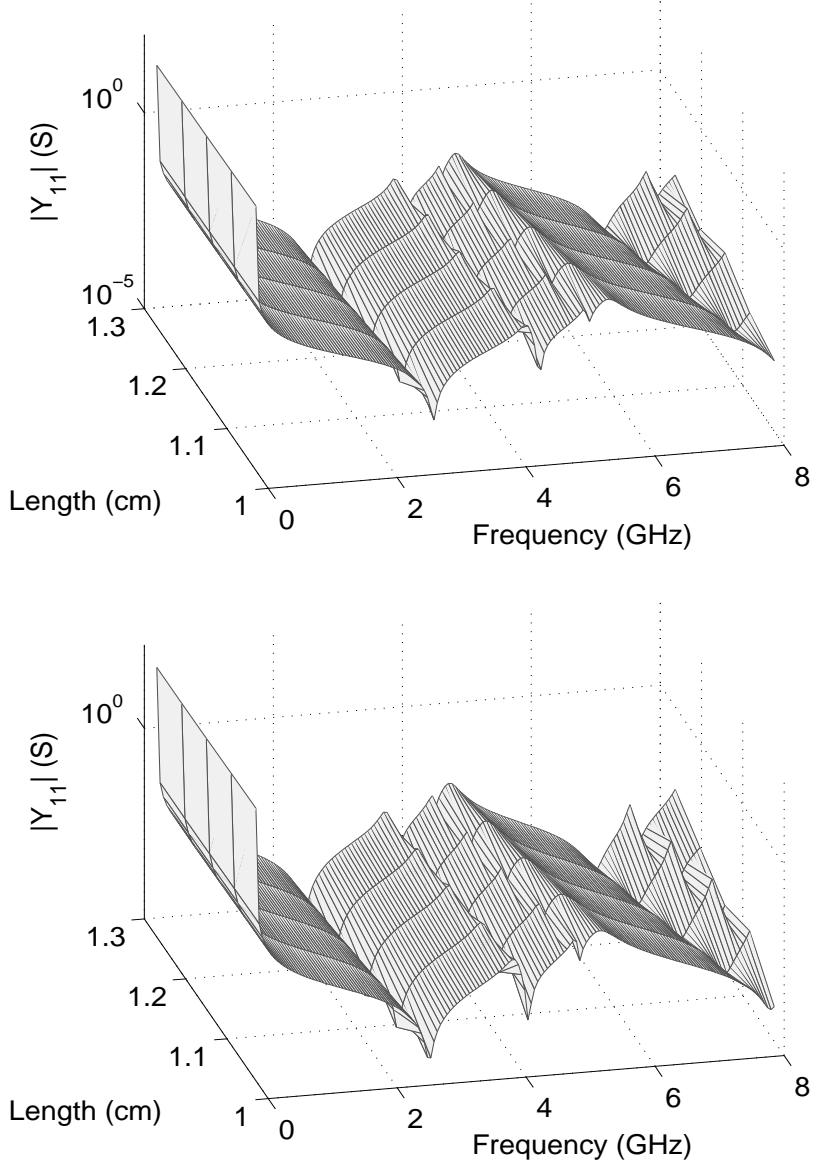

Fig. 9. Magnitude of the trivariate $\tau \mathrm{ROM}$ of $\mathbf{Y}_{11}(s, L, S)$ for $\mathrm{S}=2.56 \mathrm{~mm}$ (top) and $\mathrm{S}=2.94 \mathrm{~mm}$ (bottom).

\section{REFERENCES}

[1] R. F. Harrington, Field Computation by Moment Methods. New York: Macmillan, 1968.

[2] A. E. Ruehli, "Equivalent circuit models for three dimensional multiconductor systems," IEEE Trans. Microw. Theory Tech., vol. 22, no. 3, pp. 216-221, Mar. 1974.

[3] J. M. Jin, The Finite Element Method in Electromagnetics, 2nd ed. John Wiley and Sons, New York, 2002.

[4] L. T. Pillage and R. A. Rohrer, "Asymptotic waveform evaluation for timing analysis," IEEE Trans. Comput.-Aided Design Integr. Circuits Syst., vol. 9, no. 4, pp. 352-366, Apr. 1990.

[5] K. Gallivan, E. Grimme, P. Van Dooren, "Asymptotic waveform evaluation via a Lanczos method," Applied Math., vol. 7, no. 5, pp. 75-80, Sep. 1994.

[6] P. Feldmann and R. W. Freund, "Efficient linear circuit analysis by Padé approximation via the Lanczos process," IEEE Trans. Comput.-Aided Design Integr. Circuits Syst., vol. 14, no. 5, pp. 639-649, May 1995.

[7] A. Odabasioglu, M. Celik, and L. T. Pileggi, "PRIMA: passive reducedorder interconnect macromodeling algorithm," IEEE Trans. Comput.Aided Design Integr. Circuits Syst., vol. 17, no. 8, pp. 645-654, Aug. 1998.

[8] A. Dounavis, E. Gad, R. Achar, M. S. Nakhla, "Passive model reduction of multiport distributed interconnects," IEEE Trans. Microw. Theory Tech., vol. 48, no. 12, pp. 2325-2334, Dec. 2000.

[9] R. Achar, M. Nakhla, "Simulation of high-speed interconnects," Proceedings of the IEEE, vol. 89, no. 5, pp. 693-728, May 2001.

[10] B. Denecker, F. Olyslager, L. Knockaert, and D. De Zutter, "Generation of FDTD subcell equations by means of reduced order modeling," IEEE Trans. Antennas Propag., vol. 51, no. 8, pp. 1806-1817, Aug. 2003.

[11] N. A. Marques, M. Kamon, L. M. Silveira, J. K. White, "Generating compact, guaranteed passive reduced-order models of 3-D RLC inter- 

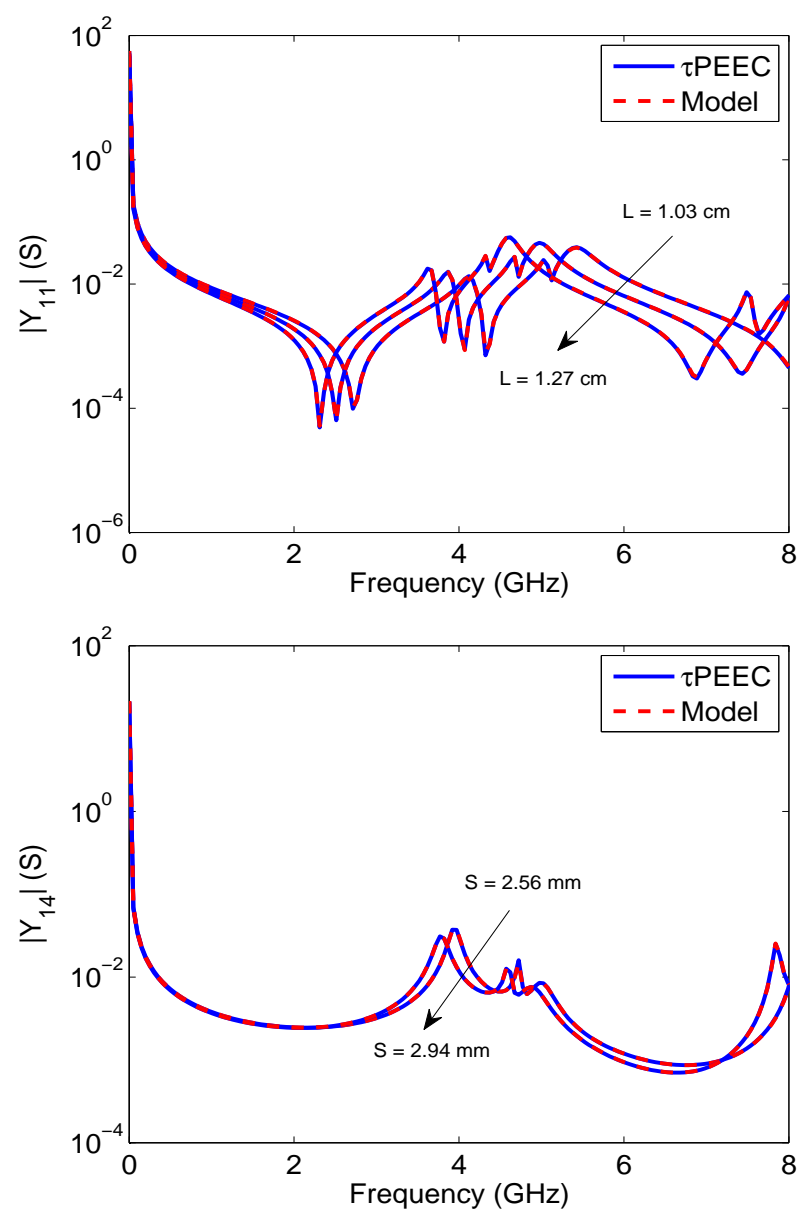

Fig. 10. Magnitude of the trivariate $\tau$ ROMs of $\mathbf{Y}_{11}(s, L, S)$ for $L=$ $\{1.03,1.15 .1 .27\} \mathrm{mm}, S=2.69 \mathrm{~mm}$ and $\mathbf{Y}_{14}(s, L, S)$ for $L=1.15 \mathrm{~mm}$, $S=\{2.56,2.94\} \mathrm{mm}$.

connects," IEEE Trans. Adv. Packag., vol. 27, no. 4, pp. 569-580, Nov. 2004.

[12] C. A. Balanis, Advanced Engineering Electromagnetics. John Wiley and Sons, New York, 1989.

[13] A. E. Ruehli and A. C. Cangellaris, "Progress in the methodologies for the electrical modeling of interconnects and electronic packages," Proc. IEEE, vol. 89, no. 5, pp. 740-771, May 2001.

[14] W. Pinello, A. C. Cangellaris, and A. Ruehli, "Hybrid electromagnetic modeling of noise interactions in packaged electronics based on the partial-element equivalent-circuit formulation," IEEE Trans. Microw. Theory Tech., vol. 45, no. 10, pp. 1889-1896, Oct. 1997.

[15] L. W. Nagel, "SPICE: A computer program to simulate semiconductor circuits," University of California, Berkeley, Electr. Res. Lab. Report ERL M520, May 1975.

[16] A. E. Ruehli, G. Antonini, J. Esch, J. Ekman, A. Mayo and A. Orlandi, "Non-orthogonal PEEC formulation for time and frequency domain EM and circuit modeling," IEEE Trans. Electromagn. Compat., vol. 45, no. 2, pp. 167-176, May 2003.

[17] P. J. Restle, A. Ruehli, S. G. Walker, G. Papadopoulos, "Full-wave PEEC time-domain for the modeling of on-chip interconnects," IEEE Transactions on Computer-Aided Design, vol. 20, no. 7, pp. 877-887, Jul. 2001.

[18] G. Antonini, J. Ekman, "On Characterizing Artifacts Observed in PEEC Based Modeling," in Proc. of the IEEE Int. Symp. on Electromagnetic Compatibility, Santa Clara, Ca, USA, Aug. 2004.

[19] A. E. Ruehli, H. Heeb, E. Bracken and R. Rohrer, "Three dimensional circuit oriented electromagnetic modeling for VLSI interconnects," in Proceedings of the Packaging, Interconnects, Optoelectronics for the Design of Parallel Computers Workshop, Mar. 1992.

[20] E. Chiprout, H. Heeb, M. Nakhla, and A. Ruehli, "Simulating 3-D retarded interconnect models using complex frequency hopping (CFH)," in Digest of Technical Papers, IEEE/ACM International Conference on Computer-Aided Design, Nov. 1993, pp. 66-72.

[21] J. R. Phillips, E. Chiprout, and D. D. Ling, "Efficient full-wave electromagnetic analysis via model-order reduction of fast integral transforms," in DAC '96: Proceedings of the 33rd annual Design Automation Conference, New York, NY, USA, 1996, pp. 377-382.

[22] J. Cullum, A. Ruehli, T. Zhang, "A method for reduced-order modeling and simulation of large interconnect circuits and its application to PEEC models with retardation," IEEE Trans. Circuits Syst. II, vol. 47, no. 4, pp. 261-373, Apr. 2000.

[23] T. Klemas, L. Daniel, and J. White, "Segregation by primary phase factors: a full-wave algorithm for model order reduction," in Design Automation Conference, 2005. Proceedings. 42nd, Jun. 2005, pp. $943-$ 946.

[24] W. Tseng, C. Chen, E. Gad, M. Nakhla, and R. Achar, "Passive order reduction for RLC circuits with delay elements," IEEE Transactions on Advanced Packaging, vol. 30, no. 4, pp. 830 -840, Nov. 2007.

[25] F. Ferranti, M. Nakhla, G. Antonini, T. Dhaene, L. Knockaert, and A. E. Ruehli, "Multipoint full-wave model order reduction for delayed PEEC models with large delays," IEEE Transactions on Electromagnetic Compatibility, accepted for publication 2011.

[26] P. K. Gunupudi, R. Khazaka, M. S. Nakhla, T. Smy, and D. Celo, "Passive parameterized time-domain macromodels for high-speed transmission-line networks," IEEE Trans. Microw. Theory Tech., vol. 51, no. 12, pp. 2347-2354, Dec. 2003.

[27] L. Daniel, O. C. Siong, L. S. Chay, K. H. Lee, and J. White, "A multiparameter moment-matching model-reduction approach for generating geometrically parameterized interconnect performance models," IEEE Trans. Comput.-Aided Design Integr. Circuits Syst., vol. 23, no. 5, pp. 678-693, May 2004.

[28] Y.-T. Li, Z. Bai, Y. Su, and X. Zeng, "Model order reduction of parameterized interconnect networks via a two-directional arnoldi process," IEEE Trans. Comput.-Aided Design Integr. Circuits Syst., vol. 27, no. 9, pp. 1571-1582, Sep. 2008.

[29] P. Gunupudi, R. Khazaka, and M. Nakhla, "Analysis of transmission line circuits using multidimensional model reduction techniques," IEEE Trans. Adv. Packag., vol. 25, no. 2, pp. 174 - 180, May 2002.

[30] M. Sampath, A. Dounavis, and R. Khazaka, "Parameterized model order reduction techniques for FEM based full wave analysis," IEEE Trans. Adv. Packag., vol. 32, no. 1, pp. 2 -12, Feb. 2009.

[31] M. Ahmadloo and A. Dounavis, "Parameterized model order reduction of electromagnetic systems using multiorder arnoldi," IEEE Trans. Adv. Packag., vol. 33, no. 4, pp. 1012 -1020, Nov. 2010.

[32] F. Ferranti, G. Antonini, T. Dhaene, and L. Knockaert, "Guaranteed passive parameterized model order reduction of the partial element equivalent circuit (PEEC) method," IEEE Transactions on Electromagnetic Compatibility, vol. 52, no. 4, pp. 974 -984, Nov. 2010.

[33] F. Ferranti, G. Antonini, T. Dhaene, L. Knockaert, and A. Ruehli, "Physics-based passivity-preserving parameterized model order reduction for PEEC circuit analysis," IEEE Transactions on Components, Packaging and Manufacturing Technology, vol. 1, no. 3, pp. $399-409$, Mar. 2011.

[34] G. Allasia, "Simultaneous interpolation and approximation by a class of multivariate positive operators," Numerical Algorithms, vol. 34, no. 2, pp. 147-158, Dec. 2003.

[35] A. E. Ruehli and H. Heeb, "Circuit models for three-dimensional geometries including dielectrics," IEEE Trans. Microw. Theory Tech., vol. 40, no. 7, pp. 1507-1516, Jul. 1992.

[36] C. Ho, A. Ruehli, P. Brennan, "The modified nodal approach to network analysis," IEEE Trans. Circuits Syst., vol. 22, no. 6, pp. 504-509, Jun. 1975.

[37] A.Weiser and S. E. Zarantonello, "A note on piecewise linear and multilinear table interpolation in many dimensions," Mathematics of Computation, vol. 50, no. 181, pp. 189-196, Jan. 1988.

[38] D. F. Watson, "Computing the n-dimensional delaunay tessellation with application to voronoi polytopes," The Computer Journal, vol. 24, no. 2, pp. 167-172, Febr. 1981.

[39] "Matlab User's Guide," The Mathworks, Inc., Natick, 2009.

[40] B. D. Anderson, S. Vongpanitlerd, Network Analysis and Synthesis. Englewood Cliffs, NJ, 1973.

[41] G. Allasia, "A class of interpolating positive linear operators: Theoretical and computational aspects," in Recent Developments in Approximation Theory, Wavelets and Applications, S. P. Singh, Ed. Dordrecht: Kluwer, 1995, pp. 1-36. 
[42] E. W. Cheney, "Multivariate approximation theory: Selected topics," in CBMS-NSF Regional Conference Series in Applied Mathematics, vol. 51. Philadelphia, PA: SIAM, 1986.

[43] R. A. Horn, C. R. Johnson, Matrix Analysis. Cambridge University Press, 1985

[44] E.-P. Li, E.-X. Liu, L.-W. Li, and M.-S. Leong, "A coupled efficient and systematic full-wave time-domain macromodeling and circuit simulation method for signal integrity analysis of high-speed interconnects," IEEE Transactions on Advanced Packaging, vol. 27, no. 1, pp. 213 - 223, Feb. 2004.

[45] R. Wang and J.-M. Jin, "A flexible time-stepping scheme for hybrid field-circuit simulation based on the extended time-domain finite element method," IEEE Transactions on Advanced Packaging, vol. 33, no. 4, pp. 769 - 776, Nov. 2010.

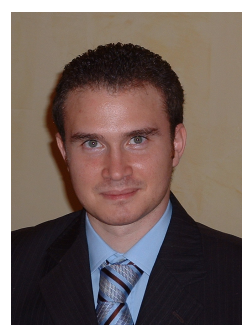

Francesco Ferranti (M'10) received the B.S. degree (summa cum laude) in electronic engineering from the Università degli Studi di Palermo, Palermo, Italy, in 2005, the M.S. degree (summa cum laude and honors) in electronic engineering from the Università degli Studi dell'Aquila, L'Aquila, Italy, in 2007, and the Ph.D. degree in electrical engineering from the University of Ghent, Ghent, Belgium, in 2011. He is currently a Post-Doctoral Research Fellow with the Department of Information Technology (INTEC), Ghent University, Ghent, Belgium. His research interests include parametric macromodeling, parameterized model order reduction, electromagnetic compatibility numerical modeling, system identification.

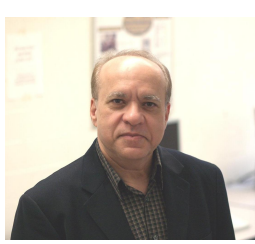

Michel S. Nakhla (S'73-M'75-SM'88-F'98) is a Chancellor's Professor of Electrical Engineering at Carleton University. He received the Ph.D. degree in Electrical Engineering from University of Waterloo, Ontario, Canada, in 1975. From 1976-88 he was with Bell-Northern Research, Ottawa, Canada, as the senior manager of the computer-aided engineering group. In 1988, he joined Carleton University, Ottawa, Canada as a professor and the holder of the Computer-Aided Engineering Senior Industrial Chair established by Bell-Northern Research and the Natural Sciences and Engineering Research Council of Canada. He is the founder of the high-speed CAD research group at Carleton University. $\mathrm{He}$ is serving on various international committees, including the standing committee of the IEEE International Signal Propagation on Interconnects Workshop (SPI), the technical program committee of the IEEE International Microwave Symposium (IMS), the technical program committee of the IEEE Conference on Electrical Performance of Electronic Packaging (EPEP) and the CAD committee (MTT-1) of the IEEE Microwave Theory and Techniques Society. He is an Associate Editor of the IEEE Transactions on Advanced Packaging and served as Associate Editor of the IEEE Transactions on Circuits and Systems. He has also served as a member of many Canadian and international government-sponsored research grants selection panels $\mathrm{He}$ serves as a technical consultant for several industrial organizations and is the principal investigator for several major sponsored research projects. His research interests include modeling and simulation of high-speed circuits and interconnects, nonlinear circuits, parallel processing, multidisciplinary optimization and neural networks.
Giulio Antonini (M'94, SM'05) received his Laurea degree (summa cum laude) in Electrical Engineering in 1994 from the Università degli Studi dell'Aquila and the Ph.D. degree in Electrical Engineering in 1998 from University of Rome "La Sapienza". Since 1998 he has been with the UAq EMC Laboratory, Department of Electrical Engineering of the University of L'Aquila where he is currently Associate Professor. His research interests focus on EMC analysis, numerical modeling and in the field of signal integrity for high-speed digital systems. He has authored or co-authored more than 170 technical papers and 2 book chapters. Furthermore, he has given keynote lectures and chaired several special sessions at international conferences. He has been the recipient of the IEEE Transactions on Electromagnetic Compatibility Best Paper Award in 1997, the CST University Publication Award in 2004, the IBM Shared University Research Award in 2004, 2005 and 2006, the IET-SMT Best Paper Award in 2008. In 2006 he has received a Technical Achievement Award from the IEEE EMC Society "for innovative contributions to computational electromagnetic on the Partial Element Equivalent Circuit (PEEC) technique for EMC applications". He holds one European Patent.

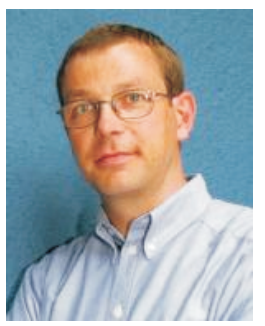

Tom Dhaene (SM'05) was born in Deinze, Belgium, on June 25, 1966. He received the Ph.D. degree in electrotechnical engineering from the University of Ghent, Ghent, Belgium, in 1993. From 1989 to 1993, he was Research Assistant at the University of Ghent, in the Department of Information Technology, where his research focused on different aspects of full-wave electro-magnetic circuit modeling, transient simulation, and time-domain characterization of high-frequency and high-speed interconnections. In 1993, he joined the EDA company Alphabit (now part of Agilent). He was one of the key developers of the planar EM simulator ADS Momentum. Since September 2000, he has been a Professor in the Department of Mathematics and Computer Science at the University of Antwerp, Antwerp, Belgium. Since October 2007, he is a Full Professor in the Department of Information Technology (INTEC) at Ghent University, Ghent, Belgium. As author or co-author, he has contributed to more than 150 peer-reviewed papers and abstracts in international conference proceedings, journals and books. He is the holder of 3 US patents.

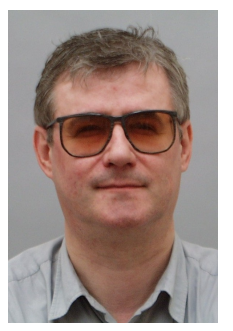

Luc Knockaert (SM'00) received the M. Sc. Degree in physical engineering, the M. Sc. Degree in telecommunications engineering and the $\mathrm{Ph}$. D. Degree in electrical engineering from Ghent University, Belgium, in 1974, 1977 and 1987, respectively. From 1979 to 1984 and from 1988 to 1995 he was working in North-South cooperation and development projects at the Universities of the Democratic Republic of the Congo and Burundi. $\mathrm{He}$ is presently affiliated with the Interdisciplinary Institute for BroadBand Technologies (www.ibbt.be) and a professor at the Dept. of Information Technology, Ghent University (www.intec.ugent.be). His current interests are the application of linear algebra and adaptive methods in signal estimation, model order reduction and computational electromagnetics. As author or co-author he has contributed to more than 100 international journal and conference publications. He is a member of MAA, SIAM and a senior member of IEEE. 


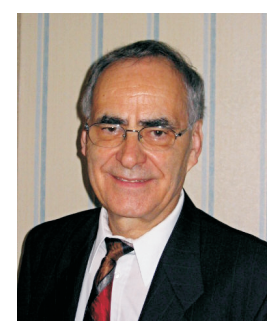

Albert E. Ruehli (LF'03) received the Ph.D. degree in electrical engineering from the University of Vermont, Burlington, in 1972, and an honorary doctorate from the Lulea University, Sweden, in 2007. He has been a member of various projects with IBM including mathematical analysis, semiconductor circuits and devices modeling, and as manager of a VLSI design and CAD group. Since 1972, he has been at IBM's T. J. Watson Research Center in Yorktown Heights, New York, where he was a Research Staff Member in the Electromagnetic Analysis Group. He is now an Emeritus of IBM Research and an adjunct professor in the EMC area at the Missouri University of Science and Technology. He is the editor of two books, Circuit Analysis, Simulation and Design (New York: North Holland 1986, 1987) and he is an author or coauthor of over 190 technical papers. Dr. Ruehli has served in numerous capacities for the IEEE. In 1984 and 1985, he was the Technical and General Chairman, respectively, of the ICCD International Conference. He has been a member of the IEEE ADCOM for the Circuit and System Society and an Associate Editor for the Transactions on Computer-Aided Design. He has given talks at universities including keynote addresses and tutorials at conferences, and has organized many sessions. He received IBM Research Division or IBM Outstanding Contribution Awards in 1975, 1978, 1982, 1995, and 2000. In 1982, he received the Guillemin-Cauer Prize Award for his work on waveform relaxation, and in 1999, he received a Golden Jubilee Medal, both from the IEEE CAS Society. In 2001, he received a Certificate of Achievement from the IEEE EMC Society for Inductance Concepts and the Partial Element Equivalent Circuit (PEEC) method. He received the 2005 Richard R Stoddart Award, and in 2007 he received the Honorary Life Member Award from the IEEE Electromagnetic Compatibility Society for outstanding technical performance. He is a member of SIAM. 